Rapid Reviews COVID-19

\title{
Review 2: "Scent dog identification of SARS- CoV-2 infections, similar across different body fluids"
}

\section{Catherine Reeve ${ }^{1}$}

${ }^{1}$ Lecturer, Queen's University Belfast, Psychology, United Kingdom

Published on: Apr 09, 2021

DOI: $10.1162 / 2 \mathrm{e} 3983 f 5 . f 8 c b 7301$

License: Creative Commons Attribution 4.0 International License (CC-BY 4.0). 


\section{$\underline{\text { RR:C19 Evidence Scale rating by reviewer: }}$}

- Reliable. The main study claims are generally justified by its methods and data. The results and conclusions are likely to be similar to the hypothetical ideal study. There are some minor caveats or limitations, but they would/do not change the major claims of the study. The study provides sufficient strength of evidence on its own that its main claims should be considered actionable, with some room for future revision.

$* * * * * * * * * * * * * * * * * * * * * * * * * * * * * * * * * * * * * * *$

\section{Review:}

In the ongoing fight to accurately detect and contain positive cases of the coronavirus, COVID-19, olfactory detection dogs ("sniffer dogs") have emerged as a highly efficient and promising detection "tool." The authors of this study reference appropriate literature to support the use of dogs as detectors of physiological changes in humans including the encouraging results of other teams that have successfully trained dogs to discriminate SARS-CoV-2-positive samples from SARS-CoV-2-negative samples in lab environments. The authors refer to a previous study of their own in which they demonstrated the ability of dogs to discriminate between tracheobronchial secretions and saliva samples from hospitalized SARS-CoV-2-positive patients (the samples had been inactivated) and SARS-CoV-2-negative samples with a high degree of sensitivity and specificity.

The current study presents an important next step in our understanding of canine detection of the virus. In this study, the authors build upon their previous work by examining whether 1 ) dogs can generalize their detection of inactivated SARS-CoV-2positive saliva samples to non-inactivated saliva samples, and 2) dogs can generalize the detection of SARS-CoV-2-positive saliva samples to urine and sweat samples. The results of the study suggest that the dogs can in fact generalize the odor of inactivated SARS-CoV-2-positive saliva samples to non-inactivated samples and urine and sweat samples with a high degree of sensitivity and specificity.

A strong aspect of the study is the experimental setup. The samples were presented to the dogs using an automated system that randomized the target sample position amongst seven potential positions, timed the duration of dogs' indications, and automatically rewarded successful indications. This removed any potential for human influence on the dogs' performance and allowed for trials to be entirely double-blind. 
Furthermore, the authors went to great lengths to ensure the safety of the researchers and the dogs, including testing the dogs for COVID-19 after each day of testing.

However, a weakness of the study is that the authors provide very few details on the progression from training to testing sessions and no details regarding what kind of samples occupied each of the seven sample positions on each trial. For example, was there 1 SARS-CoV-2-positive sample per trial with the remaining 6 positions containing SARS-CoV-2-negative samples? Alternatively, did the six positions contain control samples (the same sample collection materials devoid of the actual biological sample), or were they simply empty? These details have implications for the calculation of performance parameters as one could argue that the absence of an indication behavior on an empty hole is not a true negative.

The authors also highlight that, unlike their first study, this study included SARS-CoV-2positive samples from people that are asymptomatic or with few symptoms, as well as SARS-CoV-2-negative samples from people with other respiratory conditions. However, the reader must review the supplementary material to be made aware that only three samples were provided by people that were positive for SARS-CoV-2 yet asymptomatic, and that these samples were used during a "transfer to urine and sweat phase" that is not described anywhere. The dogs' performance with these samples is not described at any point. Only four samples were provided by people that tested SARS-CoV-2-negative but had other respiratory conditions and the results suggest that these samples were presented simultaneously alongside SARS-CoV-2-positive samples and SARS-CoV-2negative samples. Although examining the dogs' performance with these samples is not an explicit objective of the study, the study would benefit from the authors presenting these findings independently, if only descriptive, as there are currently no studies examining how dogs might perceive asymptomatic samples nor are there studies examining their ability to discriminate between COVID-19 and other respiratory conditions.

Taken together, the written presentation of this paper could be clearer, and more details on the sample presentation would allow a more thoughtful interpretation of the results. Despite these shortcomings, the results show strong evidence that the dogs generalized detection of inactivated samples to non-inactivated samples and different sample types. These findings support the idea that dogs can detect a SARS-CoV-2specific odor. This study is valuable in advancing the use of COVID-19 detection dogs as a screening tool for SARS-CoV-2 infections. 